\title{
Role of Endogenous Beta-Adrenergic Mechanism in the Pathogenesis of Spontaneous Myocardial Fibrosis in Rats
}

\author{
Shinsuke Yoshimura and Koroku Hashimoto \\ Hatano Research Institute, Food and Drug Safety Center, \\ Hadano $25 \%$
}

\begin{abstract}
Yoshimura, S. and Наshimoto, K. Role of Endogenous Beta-Adrenergic Mechanism in the Pathogenesis of Spontaneous Myocardial Fibrosis in Rats. Tohoku J. exp. Med., 1988, 155 (4), 327-333_ The role of endogenous betaadrenergic activation in the pathogenesis of spontaneous myocardial fibrosis was investigated in male and female Sprague-Dawley (SD) rats. For the purpose of blocking endogenous beta-adrenergic activities, atenolol, a beta-adrenergic blocking a agent, was given at 20,80 and $320 \mathrm{mg} / \mathrm{kg} /$ day mixed in the diet for 12 months. The transverse sections of the ventricles including the vulnerable region for spontaneous myocardial fibrosis was stained by Azan-Mallory method for collagen fibers and examined morphometrically, while being compared with the histological findings from standard hematoxylin-eosin procedure. The fibrosis was graded into 4 classes and compared between nontreated groups and treated groups of both sexes. Fibrous foci developed principally in the subendocardial myocardium. Atenolol failed to produce significant systemic toxic effects not only on weight gains and daily intakes of food but also on death rates. Atenolol did not prevent development of myocardial lesions in both sexes, whereas it significantly promoted fibrosis in the medium and high dose groups of male rats but not at any dose levels in female animals. It is suggested that endogenous beta-adrenergic activation plays no major role in the onset or development of spontaneous myocardial lesions, whereas the adrenergic system may probably control collagen synthesis in myocardial fibrosis.—rat; myocardial fibrosis; atenolol; beta-adrenergic blocking
\end{abstract}

It is well known that spontaneous myocardial lesions are common in chronic studies of rats over the age of 12 months. These were described as myocardial degeneration (Berg and Simms 1965; Simms 1967), myocarditis (Lehr 1965) and myocardial fibrosis (Fairweather 1967; Coleman et al. 1977 ; Muraoka et al. 1977 ; Hayashi 1980). These lesions start at about 7 months of age as small necrotic foci which are gradually replaced by collagen fibers. In the advanced stage fibrous regions become dominant, while necrotic foci are less evident. The characteristic location of focal disseminated myocardial necrosis shows a sharp contrast to ischemic necrosis following obstruction of the coronary artery. The occurrence of

Received March 15, 1988; revision accepted for publication June 27, 1988. 
these myocardial lesions in rats has not changed even with improved husbandry condition. Although the aetiology of spontaneous myocardial fibrosis has not yet been well elucidated, certain cardiotoxic but noncoronarogenic mechanisms induced by some endogenous factors may probably play a role in the natural history of spontaneous myocardial fibrosis.

Since the cardiotoxic response was demonstrated following administration of adrenal hormones (Nahas et al. 1958 ; Szakacs and Cannon 1958), numerous report have been published on myocardial lesions induced by catecholamines (Rona and Bier 1981). On the other hand, the regulatory mechanisms of collagen synthesis have been extensively studied both in vitro (Baum et al. 1978, 1980 ; Berg et al. 1981) and in vivo experiments (Lindenschmidt and Witschi 1985), and cyclic nucleotides have been suggested to control fibrosis via collagen synthesis.

It interested the authors to examine the chronic effects of beta-adrenergic blocking on myocardial lesions in order to confirm the speculated interplay between endogenous beta-adrenergic activation and the natural history of spontaneous myocardial fibrosis in rats.

\section{Materials and Methods}

A total of 128 four-week old SD rats composed of 64 males and 64 females were obtained from the Charles River Breeding Laboratories Japan and then quarantined for one week. Each of these SD rats was housed separately in a wire-bottom metal cage and kept in a barrier system at a temperature of $24 \pm 1^{\circ} \mathrm{C}$ and a relative humidity of $55 \pm 5 \%$. Room air was changed 15 times per hour. A $12 \mathrm{hr}$ light cycle was used. The animals were fed with commercial standard diet (CE-2, Japan Clea Co., Tokyo). Drinking water and diet were available ad libitum. The daily uptakes of food and drinking water were recorded. Weight gains were measured every four weeks. The drug used for the purpose of prolonged blocking of endogenous beta-adrenergic activities was atenolol (Sumitomo Chemicals, Osaka) which was mixed in the diet at scheduled doses of 20,80 and $320 \mathrm{mg} / \mathrm{kg} /$ day as calculated from the daily uptake of food as shown in Table 1. Sixty four male and female rats were divided in 4 groups by sex, composed of a nontreated group and 3 treated groups at 3 dose levels. Each group consisted of 16 animals. All the animals were sacrificed at 12 months and moribund and dead animals were submitted for necropsy. The heart as well as other organs and tissues were fixed in buffered $10 \%$ formalin and standard histological techniques with hematoxylin and eosin were used. The transverse paraffin section of the ventricle (Fig. 1A) was used for classification of grades of spontaneous myocardial fibrosis. Morphometrical analysis was applied in the typical patterns of fibrosis as follows: AzanMallory stained sections were projected on graph paper at a magnification of $\times 20$ and the relative areas of fibrosis in the transverse section were measured with a point counting method. The morphometrical data for myocardial fibrosis were classified from grade 0 (no change) to grade IV as shown in Fig. 1B (grade II), Fig. 1C (grade III) and Fig. 1D (grade IV).

Grade 0: No particular finding in the transverse section.

Grade I : A few small foci of fibrosis consisting of thin scattered collagen fibers.

Grade II : More numerous larger foci of fibrosis. Relative area of fibrous foci was less than $1 \%$ as shown in Fig. $1 \mathrm{~B}$.

Grade III : Foci of spreading fibrosis showing a network appearance. Relative area of fibrous foci was more than $1 \%$ but less than $5 \%$ as shown in Fig. $1 \mathrm{C}$.

Grade IV : Myocardium was widely replaced by connective tissue. A few degenerating 

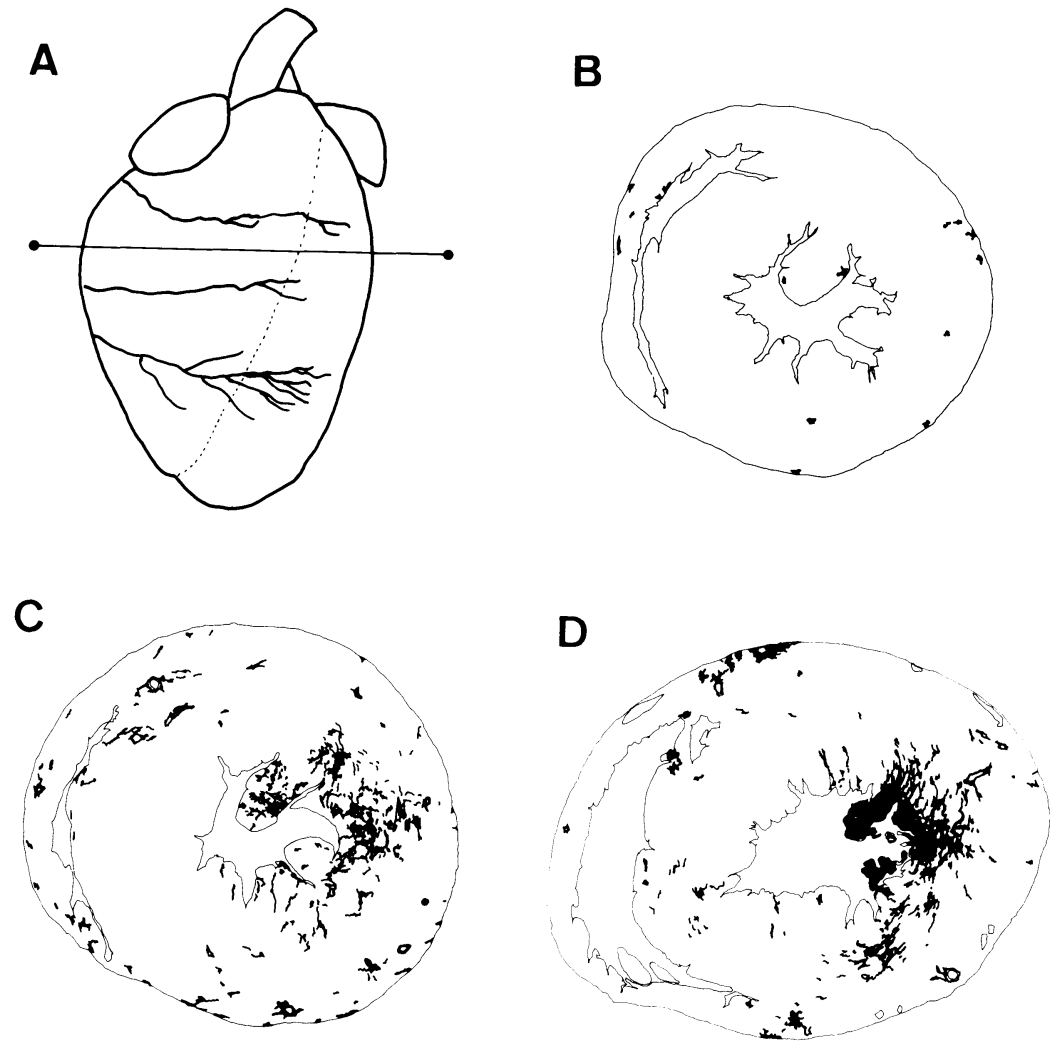

Fig. 1. Grade of fibrosis.

A, Level of transverse section of ventricles $(\bullet-\bullet)$; B, grade II fibrosis ;

C, grade III fibrosis; D, grade IV fibrosis.

myocardial cells were scattered in severely affected areas. Papillary muscles and subendocardial regions of the left ventricle were most commonly and most markedly affected. Relative area of fibrous foci was more than $5 \%$ as shown in Fig. 1D. Grade IV fibrosis was not observed in the present study.

Data were statistically analyzed by Mann-Whitney U test.

\section{Results}

Weight gains and intakes of food

Weight gains and intakes of food were not significantly different between nontreated animals and treated animals of each sex as shown in Fig. 2.

\section{Death rates}

All the animals in the nontreated groups of either sex survived for 52 weeks, while 4 male animals in total died among the lower, medium and high dose groups and 2 females in the medium dose group. The death rates were not significantly 

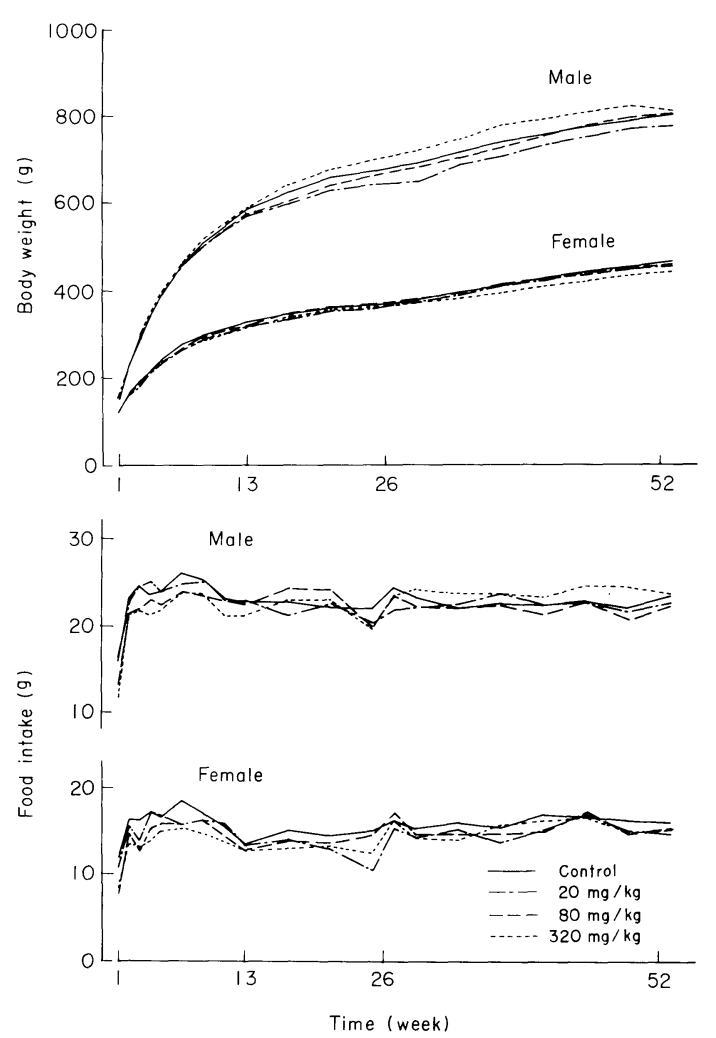

Fig. 2. Weight gains and food intake of rats with oral administration of atenolol for 52 weeks

different between the nontreated groups and treated groups in both sexes as shown in Table 1. Necropsy findings of the dead animals are given at the bottom of the table.

\section{Morphometry of myocardial fibrosis}

The transverse sections of the ventricles including the vulnerable region for spontaneous myocardial fibrosis were examined morphometrically in the specimens stained by Azan-Mallory method for collagen fibers for comparison with those stained by the standard hematoxylin-eosin method. Fibrous necrotic foci were scattered principally in the subendocardial region of the ventricular muscles including the papillary muscle and also in the subepicardial myocardium though much less were observed. In the early stage of spontaneous myocardial lesion, capillaries still remained in these necrotic foci. Phagocytes appeared in these necrotic foci hereafter, and the fibrous region became dominant while necrotic foci were less evident in the advanced stage. Fibrosis was graded into 4 classes and compared between the nontreated groups and treated groups with atenolol.

Myocardial fibrosis appeared more severely in male rats than in female 
TABLE 1. Effects of long-term blocking of endogenous beta-adrenergic activation by atenolol on spontaneous myocardial fibrosis in rats

\begin{tabular}{|c|c|c|c|c|c|c|c|c|c|}
\hline \multirow{2}{*}{ Sex } & \multirow{2}{*}{$\begin{array}{c}\text { Dose } \\
\text { (mg/kg/ } \\
\text { day) }\end{array}$} & \multicolumn{2}{|c|}{$\begin{array}{l}\text { Number of } \\
\text { animals }\end{array}$} & \multicolumn{5}{|c|}{$\begin{array}{l}\text { Grades of } \\
\text { fibrosis }\end{array}$} & \multirow{2}{*}{$\begin{array}{c}\text { Statistical } \\
\text { analysis } \\
p<\end{array}$} \\
\hline & & Initial & Survived & 0 & I & II & III & IV & \\
\hline \multirow[t]{4}{*}{ M } & Control & 16 & 16 & 5 & 4 & 7 & 0 & 0 & \\
\hline & 20 & 16 & $14^{\mathrm{a}, \mathrm{b}}$ & 4 & 4 & 7 & 1 & 0 & n.s. \\
\hline & 80 & 16 & $15^{c}$ & 0 & 1 & 13 & 2 & 0 & 0.01 \\
\hline & 320 & 16 & $15^{\mathrm{d}}$ & 0 & 3 & 10 & 3 & 0 & 0.05 \\
\hline \multirow[t]{4}{*}{$\mathrm{F}$} & Control & 16 & 16 & 7 & 6 & 3 & 0 & 0 & \\
\hline & 20 & 16 & $15^{\mathrm{e}}$ & 10 & 5 & 1 & 0 & 0 & n.s. \\
\hline & 80 & 16 & $15^{\mathrm{f}}$ & 10 & 4 & 1 & 1 & 0 & n.s. \\
\hline & 320 & 16 & 16 & 11 & 3 & 2 & 0 & 0 & n.s. \\
\hline
\end{tabular}

a, Fibrosarcoma of the heart.

b. Nephroblastoma with metastases to the lung.

c. Congestion of the liver and lung.

d, Right ventricular fibrosis and pulmonary edema.

e, Pituitary adenoma.

f. Fibrosarcoma of the subcutaneous with metastases to the heart, adrenal and bone marrow. Nephroblastoma.

n.s., not significant.

animals as shown in Table 1. The incidence and severity of fibrosis were significantly prominent in the medium and high dose groups of atenolol in male rats, but not different between nontreated and treated female animals.

\section{Discussion}

Chronic administration of atenolol at doses of 20,80 and $320 \mathrm{mg} / \mathrm{kg} /$ day for 12 months failed to induce toxic signs in both weight gains and daily intakes of food and drinking water. The death rates were also not significantly different between nontreated groups and treated groups, although necropsy of few dead animals showed signs of cardiovascular injury in both sexes among the treated groups.

Szakacs and Cannon (1958) first demonstrated $l$-norepinephrine myocarditis, and Nahas et al. (1958) reported on the morphological changes following administration of adrenal hormones. Raab et al. (1961) pioneered the studies of catecholamine-induced cardiovascular pathology in SD rats. He proposed that stress resulted in overstimulation of the sympathetics which in turn disproportionately increased oxygen consumption. Consequently, myocardial hypoxia ensued which then led to myocardial degeneration. Since the present authors had speculated interplay between endogenous beta-adrenergic activation and the natural history of spontaneous myocardial fibrosis in rats from the characteristic 
location of myocardial lesions, it was rather surprising to find no evidence for the protection of spontaneous occurrence of myocardial lesion by prolonged blocking of endogenous beta-adrenergic stimulation in rats of both sexes. Furthermore, it was evident that myocardial fibrosis developed more frequently and more severely in male rat groups treated with the medium and high doses of atenolol.

Previous studies have shown that collagen synthesis by fibroblasts from various tissues was influenced by various factors. Among them, Baum et al. $(1978,1980)$ first demonstrated selective decreases in collagen production due to elevated cAMP levels in culture of human fibroblasts in the presence of prostaglandin E2 and cholera toxin. Berg et al. (1981) observed the suppression of collagen production in human lung fibroblast culture and the increase in intracellular cAMP content in the presence of isoproterenol which was effectively antagonized by $l$-propranolol. Since collagen production is sensitive to the small changes of cAMP content in cultured cells, he suggested that beta-adrenergic stimulation is an important mechanism for the regulation of collagen production in the body. Recently Lindenschmidt and Witschi (1985) reported propranolol-induced increases of pulmonary collagen in male mice after acute pulmonary injuries due to butylated hydroxytoluene and bleomycin with inhalation of high concentrations of oxygen, whereas a significant decrease of cAMP and increase of cGMP with a consequent decrease in the $\mathrm{cAMP} / \mathrm{cGMP}$ ratio were demonstrated. However, there was no elevation in pulmonary collagen by propranolol when given to undamaged lung.

The observations of the present study suggest that beta-adrenergic stimulation does not play a major role in the onset or development of spontaneous myocardial lesions, whereas adrenergic system may probably controls collagen synthesis in these lesions by activation of the adenylate cyclase-cAMP mechanism. It is well known that the development of fibrosis is the result of complex multifactorial mechanisms, which mediate the characteristic responses attributed to many hormones. Myocardial fibrosis is more frequent and severe in male rats as confirmed in many studies cited above. This in turn suggests endogenous androgen of male sex hormone acting as one of facilitatory factors for collagen synthesis which is regulated by the adrenergic system. This may be a probable reason why blocking of endogenous beta-adrenergic activities resulted in promotion of myocardial fibrosis in male rats alone but not in female animals. Further studies, however, are necessary for elucidation of the original mechanism of spontaneous myocardial injuries in rats.

\section{Acknowledgments}

We thank Dr. Yuzo Hayashi, Director of the Division of Pathology, National Institute of Hygienic Science for his advice in the histological examination. 


\section{References}

1) Baum, B.J., Moss, J., Breul, S.D. \& Crystal, R.G. (1978) Association in normal human fibroblasts of elevated levels of adenosine $3^{\prime}: 5^{\prime}$-monophosphate with a selective decrease in collagen production. J. biol. Chem., 253, 3391-3394.

2) Baum, B.J., Moss, J., Breul, S.D., Berg, R.A. \& Crystal, R.G. (1980) Effect of cyclic AMP on the intracellular degradation of newly synthesized collagen. J. biol. Chem., 255, 2843-2847.

3) Berg, B.N. \& Simms, H.S. (1965) Neutrition, onset of disease, and longevity in the rat. Canad. med. Ass. J., 93, 911-913.

4) Berg, R.A., Moss, J., Baum, B.J. \& Crystal, R.G. (1981) Regulation of collagen production by the beta-adrenergic system. J. clin. Invest., 67, 1457-1462.

5) Coleman, G.L., Barthold, S.W., Osbaldiston, G.W., Foster, S.J. \& Jonas, A.M. (1977) Pathological changes during aging in barrier-reared Fischer 344 male rats. J . Geront., 32, 258-278.

6) Fairweather, F.A. (1967) Cardiovascular disease in rats. In : Pathology of Laboratory Rats and Mice, edited by E. Cotchin \& F.J.C. Roe, Blackwell Sci. Pub., Oxford, pp. $213-227$.

7) Hayashi, Y. (1980) Report from the working group on long-term holding of experimental animals. Exp. Anim., 29, 192-197. (Japanese)

8) Lehr, D. (1965) Lesions of the cardiovascular system. In: The Pathology of Laboratory Animals, edited by W.E. Ribelin \& J.R. McCoy, C.C. Thomas, Springfield, pp. 124-159.

9) Lindenschmidt, R.C. \& Witschi, H.P. (1985) Propranolol-induced elevation of pulmonary collagen. J. Pharmacol. exp. Ther., 232, 346-350.

10) Muraoka, Y., Itoh, M., Maeda, Y. \& Hayashi, Y. (1977) Histological changes of various organs in aged SD-JCL rats. Exp. Anim., 26, 1-12. (in Japanese with English abstract)

11) Nahas, G.G., Brunson, J.G., King, W.M. \& Cavert, H.M. (1958) Functional and morphological changes in heart lung preparation following administration of adrenal hormones. Amer. J. Path., 34, 717-729.

12) Raab, W., Stark, E., MacMilan, W.H. \& Gigee, W.R. (1961) Sympathogenic origin and antiadrenergic prevention of stress-induced myocardial lesions. Amer. J. Cardiol., 8, 203-211.

13) Rona, G. \& Bier, C. (1981) The role of coronary no-flow, reflow phenomenon in myocardial injury. In : Cardiac Toxicology, Vol. 1, CRC Press, Florida, pp. 159-178.

14) Simms, H.S. (1967) Longevity studies in rats. I. Relation between life span and age of onset of specific lesions. In : Pathology of Laboratory Rats and Mice, edited by E. Cotchin \& F.J.C. Roe, Blackwell Sci. Pub., Oxford, pp. 733-747.

15) Szakacs, J.E. \& Cannon, A. (1958) l-Norepinephrine myocarditis. Amer. J. clin. Path., 30, 425-434. 